\title{
Una mirada al devenir de la didáctica docente. Investigación horizontes de la praxis didáctica ${ }^{1}$
}

\author{
Flor Elisa Giraldo Ardila ${ }^{2}$
}

\begin{abstract}
Resumen
El presente artículo deviene del ejercicio didáctico realizado con algunos docentes del municipio de Marmato en el departamento de Caldas, en el cual a través de las entrevistas aplicadas a algunos maestros de la población, se construyeron las didactobiografias, que enriquecieron este proceso, donde los protagonistas son dos, los peritos versados en educación, y los estudiantes, razón de ser de la educación.

Es así, como después de revisar el camino de aquellas personas versadas en el tema de la educación, ahora se hace un alto para realizar una mirada en el entorno próximo, en el ámbito de la formación, de la enseñanza, para contemplar la manera en que los docentes desarrollan el arte de ser maestros, de allí entonces, esta contemplación al acontecer de la formación didáctica, como entrenamiento fundamental que garantiza el éxito en el aula.

Palabras clave: Horizontes, didáctica, trayecto, deyecto, proyecto, praxis, educación, docentes, estudiante, didactic - biographic, avances tecnológicos.
\end{abstract}

\section{A look at the future of the didactic teaching. Horizons of didactic practice}

\section{Summary}

This article becomes the teaching exercise conducted with some teachers in the municipality of Marmato in the department of Caldas, which through interviews applied to some teachers of the population, were built didactic - biographic, which enriched this process, where the protagonists are two, experts versed in education, and students, raison to be of the education.

So, and after reviewing the path of those versed in the subject of education, now becomes a high to perform a look in the immediate environment in the area of training, education, to contemplate how that teachers develop the art of being teachers, then from there, this contemplation the events of didactic training, training as essential to guarantee your success in the classroom. Keywords: Horizons, didactic way, deyecto, project, practice, education, teacher, student, didactic - biographic, technological advances.

1 Recibido: noviembre 18 del 2008. Aprobado enero 15 del 2009.

2 Nacionalidad, colombiana. Docente, ponente en eventos nacionales. Magister en Educación-Docencia de la Universidad de Manizales. 


\section{Introducción}

\section{“Quien volviendo a hacer el camino viejo aprende el nuevo, puede considerarse un maestro".}

Confucio.

Cuando se ejerce el maravilloso oficio de ser maestra, cuando se anhela con todo el corazón compartir y transmitir el conocimiento a las nuevas generaciones, cuando el corazón late aprisa ante la urgencia de enseñar en estos tiempos modernos cada vez más necesitados y acelerados.

Cuando se siente el serio compromiso y la responsabilidad de haber elegido una profesión por medio de la cual se convierte en formadores de niños y niñas de bien y cuando se pretende cumplir con el deber de la pedagogía como un saber fundante en la educación, entonces la mente y los ojos, se vuelven para hacer un recorrido por aquellas teorías y aquellos expertos, pedagogos, sicólogos y educadores que han marcado el camino a través de estrategias especificas, que van desde los métodos tradicionales hasta los más modernos, para determinar cuáles han sido sus herramientas, sus estrategias, en el campo de la didáctica y la docencia.

Es así, como después de revisar el camino de aquellos, ahora se hace un alto para realizar una mirada en el entorno próximo, en el ámbito de la educación, seleccionando un grupo de maestros del eje cafetero, para realizar el ejercicio conocido como DIDACTOBIOGRAFIA, para contemplar la manera en que ejecutan el arte de ser maestros, de allí entonces, esta mirada al acontecer de la formación didáctica, como entrenamiento fundamental que garantiza el éxito en el aula.

La evocación de la didáctica docente, implica hacer memoria del acontecer de la didáctica en general, significa dar una mirada a la forma como los educadores están preparando a los niños y niñas de hoy, para que sean grandes hombres y mujeres el mañana, que en su momento serán los encargados de formar y ser ejemplos idóneos para las próximas generaciones.

Se ha escrito tanto, se ha hablado, se ha especulado, se han tejido tantas definiciones acerca de la didáctica, como términos derivados de ella, que considero necesario, antes que nada procurar hacer un acercamiento a las voces de los expertos para determinar realmente que es la didáctica, como se verá a lo largo de estas líneas, que a su vez irán conformando párrafos construidos con las voces de expertos, algunos muy conocidos, otros muy valiosos para sus regiones y para sus estudiantes, como los que se tomaron para realizar las Didactobiobrafias, de las cuales se hará mención en posteriores renglones.

Antes de mencionar estos términos derivados de la didáctica, y hablar sobre los encargados de ponerla en práctica, es pertinente tener plena conciencia, clara cognición de lo que es realmente la didáctica. "Se define como la disciplina científico-pedagógica que tiene como objeto de estudio los procesos y elementos existentes en la materia en sí y el aprendizaje. Es, por tanto, según Herrera, "la parte de la pedagogía que se ocupa de los sistemas y métodos prácticos de enseñanza destinados a plasmar en la realidad las directrices de las teorías pedagógicas"3 (Herrera Fuentes 2004)

La didáctica de la mano de algunos componentes pedagógicos, así como la estructura escolar y la orientación educativa, intenta fundamentar y regular los procesos de enseñanza - aprendizaje.

A continuación se nombran algunos de los componentes que actúan en el acto didáctico

- el docente o profesor

- el discente o alumno

3 Herrera Fuentes, Jorge Luis, La didáctica del proceso docente para el desarrollo de la práctica laboral en las empresas, 2004 


\section{Plumilla Educatival}

- el contexto social del aprendizaje

- el curriculum

El currículum es un sistema de vertebración institucional de los procesos de enseñanza - aprendizaje que tiene fundamentalmente cuatro elementos constitutivos: objetivos, contenidos, metodología y evaluación.

La didáctica siempre lleva implícitos estos elementos, los cuales son definidos, entendidos, aplicados de forma general de acuerdo a los lineamientos de la educación y en forma particular conforme al contexto, al momento, a las condiciones especificas, al entorno que se esté viviendo, al marco en el cual se este desenvolviendo esta practica educativa.

De igual manera, la didáctica es un indagar para conocer si los educandos se están formando teniendo en cuenta sus intereses, necesidades y exigencias de los tiempos actuales. Para esto es primordial hacer un recorrido en trayecto - deyecto - proyecto, "proponer una lectura crítica de los límites del saber y del saber transmitir o enseñar"4 (González, 2008). Retomando esta frase, se puede ciertamente comparar con la profundidad de los pensamientos de Dante, al expresar: "No menos que saber me gusta dudar" (Dante Alighiere); pues es allí, en la duda, donde nace el interrogante que nos conduce al saber, que lleva al conocimiento, porque ¿que sería de la práctica de la didáctica sin la profundidad del saber del maestro?

Otra bella definición, de un maestro de la región y que hace parte de la didactobiografia realizada en Marmato Caldas, reza de la siguiente manera, "es la forma específica de alcanzar los logros de acuerdo a la naturaleza de las astucias que el maestro debe implementar para lograr su cometido y que tiene que ver

4 González González, Miguel investigador principal, Cano, Cardona, Giraldo coinvestigadores, Investigación "Horizontes de la Praxis Didáctica" 2008, Universidad de Manizales. con la heterogeneidad de sus alumnos y el contexto situacional de la comunidad educativa". (Zapata, 2008) El concepto de José Enver Ayala Zuluaga, 2006, (p16) pone a la DIDACTICA como un elemento fundamental para lograr la denominada motivación, con ella, será más fácil enseñar a reflexionar, al leer, a escribir , a comunicar , a criticar, a discutir, a realizar procedimientos; a partir de una buena estrategia didáctica se puede generar una seducción que conlleve aprendizajes posteriores. (González, 2008).

\section{El ejemplar ejercicio de la didáctica}

“La buena didáctica es aquella que deja que el pensamiento del otro no se interrumpa y que le permite, sin notarlo, ir tomando buena dirección".

\section{Enrique Tierno Galván.}

Encaminar, es sin lugar a dudas uno de los propósitos de la didáctica del docente, guiar, conducir, dirigir, motivar al estudiante dejándole alas para volar, es dar tal educación que no se impida el pensamiento del otro. El estudiante del presente requiere una didáctica activa, articulada a los avances científicos y tecnológicos, siendo notables los aprendizajes alcanzados a través de estos medios audiovisuales e interactivos, de ahí la importancia del computador, el internet, el video beam, la televisión, la grabadora y otros, que si son usados de una manera eficiente, pueden optimizar la educación, convirtiéndose realmente en ayudas didácticas valiosas para los docentes; utilizar apropiadamente estos recursos, hace parte de la motivación que cada docente debe tener en su práctica diaria.

Con el tema de la didáctica se pretende explorar sobre la forma como los maestros mantienen a los estudiantes estimulados, cautivados e interesados por la adquisición de nuevos conocimientos que les permita un verdadero crecimiento integral, que 
favorezca sus relaciones con el entorno y sociedad. Encontrar testimonios sobre la forma como se desarrollan habilidades de liderazgo, democracia y respeto, la manera como se estimulan los estudiantes para llegar a ser hombres críticos, reflexivos y situados; reconocer las didácticas empleadas en el proceso de formación de sujetos, que posibilite un ser, un saber y un actuar como líder de su propia existencia y como nuevo ciudadano del mundo.

Al hablar de la importancia de la didáctica, de su trascendencia en la actualidad para formar estos ciudadanos del mundo, es necesario mirar atrás y en ese acontecer, como un recorrido por la historia, el pasado, el presente y aun una mirada visionaria del futuro, como aquello que puede venir, que puede acontecer, que puede suceder se estría hablando del devenir de la didáctica, el cual es un recordar cómo ha sido la educación en tiempos pasados para retomar lo bueno, es un autoreflexionar sobre como se trabaja en la actualidad y qué se pretende, hacia donde se quiere llegar. Es un preparar al individuo para valerse por si mismo en la vida; como expresa Estela Quintar 2006, p27 "Es un resignificar, acto que solo se logra a través de la autorreflexión de lo que somos y de porqué somos lo que somos, lo cual nos impulsará a comprender, aprehender y encontrarnos activamente en el presente y en la construcción propositiva de proyectos de vida individual y social, libertarios y democráticos" 5 .

\section{El amor por la experiencia docente}

\section{"La felicidad no está en hacer lo que uno quiere, sino en querer lo que uno hace".}

Jean Paul Sartre.

5 Quintar, Estela B, La Enseñanza como puente a la vida. Colección Conversaciones Didácticas, Instituto Pensamiento y Cultura En América Latina, A.C. México. 2006.
Al trabajar sobre el tema de la didáctica se pudo constatar que ésta es una época de grandes cambios y avances tecnológicos, donde se hace necesario contar con docentes capacitados e interesados por brindar a sus educandos una formación acorde con sus exigencias y las de la época; época en la cual los jóvenes son más activos y hábiles para el manejo de dichas tecnologías, pero a su vez más apáticos por los aprendizajes dentro del aula con métodos tradicionales y memorísticos.

Considerando que el aprendizaje es fundamental en el desarrollo de la especie humana, ya que es la forma por el cual cada individuo evoluciona, por él la especie se continúa en el acontecer de las civilizaciones. Teniendo en cuenta que hay aprendizajes de hábitos, de contenidos, de conductas, de normas y en la medida que el sujeto es capaz de ir poniendo a su servicio, al servicio de su familia, de su comunidad todos esos aspectos cognitivos y de comportamiento, adquiridos en la escuela al lado de sus maestros podrá resignificar sus aprendizajes, su sentido de vida.

La educación es un transcurso, una carrera, es el proceso por medio del cual el hombre tiene la capacidad de desarrollar habilidades y destrezas, de vivenciar valores, de reflexionar y dar sentido a lo que hace, a la forma cómo se relaciona y qué valores otorga a las cosas con las cuales se rodea, es trascendental y necesario que los docentes aprovechen al máximo esta grandiosa labor para formar los hombres líderes, erguidos y situados" 6 (González, 2008), requeridos por esta sociedad tan abatida por tanto conflicto político, social, económico... sociedad que necesita de personas que luchen por un bien común, y no solo de personas que tengan como propósito ¡el poder i en especial el económico

6 Idem, página 4 
Todo docente tiene como misión desarrollar en el ser humano ciertas capacidades para actuar dentro de la sociedad, transformando y transformándose en una interrelación que documenta su historia. Para satisfacer sus necesidades. Como Io indica Manuel Argumedo, 1986, "Educar es una actividad dirigida a transformar las circunstancias a través de la transformación de los sujetos, interviniendo en sus procesos de aprendizaje." Para educar es preciso, entonces, definir en qué sentido se pretende que los sujetos se transformen y cómo se ha de intervenir en el aprendizaje o, dicho de otra manera, qué orientación se pretende dar a los procesos de aprendizaje"'. Quintar, Estela B, (2006)

Es substancial despuntar que cuando se tiene el compromiso de ser maestro, la labor nunca termina, asiduamente se piensa y se trabaja en función de los estudiantes y la manera de sacarlos adelante con buenas bases que les permita si no a todos, al menos a la mayoría de ellos, ser aceptados en su comunidad y sociedad. El niño necesita que haya adultos que le ayuden a estabilizar progresivamente las capacidades mentales que le ayudaran a vivir en el mundo, a adaptarse a las dificultades con que se encuentre y a construir él mismo, progresivamente sus propios saberes" Frankenstein Educador, (1998); es decir que con la educación recibida por el ser humano en los distintos campos de su que hacer cultural, éste logra ubicarse y definirse, en otras palabras, logra dar sentido a su vida; convirtiendo la educación y los educadores en una de las influencias más importantes en la vida y el desarrollo de los niños, jóvenes e incluso adultos para la formación de las nuevas generaciones.

7 Idem, página 6

\section{La perseverancia conlleva a la genialidad}

"El genio comienza las grandes obras, pero sólo el trabajo las acaba".

Joseph Joubert.

Es realmente maravilloso observar como el amor y la pasión por la docencia, por la habilidad de enseñar, se convierte en el compromiso que sella las grandes obras. Así es, pues los docentes no se forman exclusivamente en las aulas de una institución educativa, ya que estos también se forman en su praxis docente, es decir, en el trabajo día a día con los estudiantes que pasan año tras año por sus manos y que dejan infinidad de enseñanzas, inquietudes y reflexiones sobre el modo más apropiado de desempeñar su labor; por ejemplo, los alumnos enseñan al docente que todos ellos son seres heterogéneos, con limitaciones, fortalezas y capacidades diferentes, por lo cual se deben tratar desde esa perspectiva, de características particulares de cada uno de los estudiantes.

A medida que el niño va creciendo y se va haciendo más independiente, va mostrando inclinación por ciertos gustos e intereses que en muchas ocasiones terminan por desviarlos de la formación recibida o de los intereses de formación trazados por los maestros o adultos, sin que esto indique que sea una persona en especial la responsable de dichos cambios; es en general toda la sociedad y el mismo ambiente vivido por el niño los responsables de ello.

A este respecto, Philippe Meirieu (1998) expresa "El niño necesita que haya adultos que le ayuden a estabilizar progresivamente las capacidades mentales que le ayudaran a vivir en el mundo, a adaptarse a las dificultades con que se encuentre y a construir él mismo, progresivamente sus propio saberes". Realmente, el compromiso con los niños es cada vez mayor, pues los retos del mundo requieren de docentes dedicados a 
su labor, amantes de su trabajo, y, por supuesto de los niños, para que comprendan a plenitud que son sus guías, que son los encargados de descubrir sus habilidades, que deben prepararlos para enfrentarse cada día a las dificultades, a llevar emocional e intelectualmente los fracasos y angustias o los triunfos obtenidos. De igual manera es el docente quien lidera los procesos de aprendizaje significativos donde el niño descubre y se apropia de los saberes.

Retomando las entrevistas realizadas en las didactobiografias, comenta el docente entrevistado, "En los salones de clase los protagonistas no son los docentes porque creen sabérselo todo, sino los estudiantes que se están preparando para afrontar el porvenir". Los estudiantes indican, continua el docente, que es necesario darles mucha participación, darles la oportunidad de expresarse, indagar y equivocarse para madurar y adquirir mejores aprendizajes; convirtiendo así al docente en un líder constructor de sociedad, el hacedor de poetas, doctores, gobernantes, empresarios... quienes finalmente serán la prueba fehaciente, concreta, palpable de las enseñanzas recibidas de un maestro. El producto final es responsabilidad de la persona porque en últimas es el propio individuo quién decide que hacer, que camino coger y cómo explotar todo su potencial de aprendizaje.

Es así como en esta época en la cual se convive con las evoluciones aceleradas de nuestra sociedad: un mundo globalizado por la economía, por la sociedad de consumo, relacionados con todo el mundo a través de la telefonía digital, el internet y demás tecnologías de comunicación, donde el profesor que se determina por la transmisión de contenidos es figura del pasado; así entonces, es necesario que los docentes se preparen pedagógicamente para impulsar un aprendizaje creativo, dado que cada uno tiene que asumir su permanente actualización en conocimientos y actitudes ante las nuevas condiciones de vida.
Todo lo anterior indica que se hace obligatorio considerar el protagonismo del niño y el joven en el proceso de aprendizaje, implementar nuevas formas de organizar el aula, a fin de que se pueda aprender juntos, respetando las diferencias. En este proceso se hace evidente que terminó la vigencia del esquema didáctico (enseñanza), del decir lo qué y cómo aprender, al de la exploración de nuevos conocimientos y de nuevos valores.

Es así como en el desarrollo de la Investigación Horizontes de La Praxis Didáctica se llevaron a cabo unos encuentros con varios docentes del eje cafetero, específicamente en el municipio de Marmato, y Manizales a quienes se les aplicó una entrevista para indagar, descubrir, contemplar la didáctica empleada en el desarrollo de las diferentes clases y actividades con los estudiantes, construyendo de esta manera el ejercicio pedagógico conocido como DIDACTOBIOGRAFÍA, de allí entonces, esta mirada al acontecer de la formación didáctica, como entrenamiento fundamental que garantiza el éxito en el aula.

Al realizar este proceso, los docentes declararon que en el nivel educativo se presentan algunas falencias o dificultades, provenientes del mismo sistema educativo, dificultades que los profesores tienen que sortear y saber manejar para alcanzar los logros propuestos o las metas señaladas para cada grupo de estudiantes, igualmente, se consiguen muchas alegrías y satisfacciones en el desempeño de esta labor; también expresan que en las aulas se cuenta con estudiantes de todas las condiciones, razón por la cual los docentes deben estar preparados para enfrentar los retos que cada día trae consigo en el diario transcurrir de la docencia para lograr con sus enseñanzas favorecer el desarrollo integral de sus estudiantes.

A continuación se escriben algunos apartes de la información suministrada por los maestros a lo largo del ejercicio ditactobiografico: 


\section{Plumilla Educativan}

\section{Dificultades educativas}

- El actual sistema educativo traza políticas homogenizantes, no tiene en cuenta las características sociales, económicas y políticas de las comunidades, se proyectan logros nacionales, sin tener en cuenta las características regionales, ni se tiene en cuenta la multipluralidad étnica de los educandos, haciendo que existan desfases en los programas educativos impuestos por el gobierno.

- La poca ayuda por parte de los adultos que rodean al niño, sobre orientaciones y normas que estimulen su sano desarrollo, hace que muchos de estos pequeños lleguen a las instituciones educativas con comportamientos antiestéticos como lo son la indisciplina, la intolerancia, la grosería, el irrespeto por los demás, generando ambientes tensos y poco aptos para el aprendizaje.

- Para investigar más allá del aula, se requiere mirar el entorno del estudiante, su familia y la comunidad en general, en ocasiones esta labor es difícil, ya que por respeto a la intimidad de las familias hay que cohibirse de indagar sobre ellas, para evitar problemas con las mismas.

- El gobierno en su afán por cumplir con algunos requerimientos económicos habla de calidad de la educación, siendo estos parámetros económicos en educación realmente las limitantes en cuanto a implementación de planta física, recursos humanos, tecnológicos y en este momento el hacinamiento permanente en los colegios, lo cual impide que realmente la educación sea de calidad; entre estos conceptos de calidad se encuentran también las reformas educativas que en lugar de beneficiar al estudiante lo perjudica al ser demasiado laxos con ellos.

- La deserción escolar, el alejamiento de las aulas de la razón de ser del maestro ELESTUDIANTE", quienes se retiran de las instituciones por diversas razones, entre ellas dificultades económicas, cambio de domicilio o académicas.

- La situación de los niños desplazados es muy difícil, ya que se violan sus derechos fundamentales, no tienen derecho a salud, ni educación y en muchas ocasiones ni a la familia; están expuestos a la violencia, a problemas sociales como el abandono, la desnutrición, la prostitución, por nombrar algunos; por lo cual se requiere que tanto el gobierno como las instituciones educativas busquen alternativas para solucionar esta problemática.

- El poco interés o quizás el temor de algunos docentes por enfrentarse al cambio, razón por la cual continúan con sus clases tradicionales, clases que provocan en los estudiantes tanta apatía por el estudio llegando a la deserción.

\subsection{Los actos de enseñanza que movilizan un interés práctico ético por el medio ambiente}

- A partir de la realidad del estudiante y salidas pedagógicas se orienta el tema del medio ambiente en clase, logrando que el estudiante evidencie la forma como se deteriora el entorno por causas naturales o por culpa de la mano del hombre, reflexionando y planteando algunas formas de solucionar la problemática vivida, así el educando crece culturalmente.

- A los estudiantes se les debe sensibilizar sobre la importancia del medio ambiente y la forma como se puede proteger este maravilloso recurso tan necesario para la vida de todos los seres vivos y tan maltratado por todos; en clase se hacen campañas de orden y aseo, se hacen actividades de siembra de árboles y plantas, incluyendo el cuidado de los mismos por parte del estudiante, se realizan talleres de dibujo y cuento cuyo tema central es el cuidado de la naturaleza. 


\subsection{Eventos didácticos que intentan configurar y prospectar un sujeto situado y erguido}

- Se debe dar gran importancia a la lúdica, ya que por medio de ésta se logra aumentar el interés por el conocimiento; el estudiante aprende a convivir a partir de valores individuales y colectivos, comprende y transforma su mundo, a la vez que se forma una comunidad escolar sensible, crítica y solidaria.

- Los hombres líderes, pensantes y situados se empiezan a formar desde el hogar, se descubren y estimulan en las escuelas al darles participación en las diferentes actividades programadas en la institución, al hacer parte del gobierno escolar y estudiantil donde tiene que ser persona activa, participativa, reflexiva, dinámica con deseos de ayudar y resaltar al grupo o comunidad que representa.

- La educación no tiene que estar proyectada a lo económico, por lo tanto los niños no se deben dejar involucrar al mercado laboral, antes de haber recibido una excelente capacitación y orientación desde las aulas. Primero se les debe permitir manifestar su sensibilidad, su capacidad de asombro, su derecho a soñar un mundo mejor.

- Es fundamental el fortalecimiento de los principios éticos y morales, es decir, se debe poner en práctica aquellas reglas o normas que se han inculcado en hogares y colegios desde los primeros años de vida del niño, con el fin de que aprendan a actuar con responsabilidad ante todo acto de la vida.

- Se debe brindar espacios de mucha participación al estudiante para que proponga y ponga en juego su creatividad, brindando así en el salón de clase espacios de opción y concertación, donde el niño se siente aceptado, escuchado e importante, afianzando además su autoestima, y motivando el espíritu de liderazgo.
- Con la pedagogía empleada se ha aportado al estudiante elementos ideológicos y de formación que han contribuido a que sean ciudadanos democráticos, respetuosos, responsables, capaces de interactuar en una sociedad difícil y cambiante ante las exigencias mundiales de globalización.

\section{El docente por venir}

- El docente en su trabajo hace lo posible por interpretar algunas actitudes, gustos o intereses de los educandos con el fin de mejorar la relación maestro-estudiante.

- Para que la práctica cotidiana trascien$\mathrm{da}$, el maestro tiene que revestirse de mucha paciencia para interpretar el comportamiento de los niños y adolecentes, de esta manera los resultados trascenderán, los cambios se notarán en el futuro.

- La universidad no es el único medio de capacitación, esta también se adquiere, maestro a maestro, recopilando la información de cómo lograron la orientación de jóvenes con problemas de comportamiento, cómo han logrado enseñar a leer con grupos tan numerosos, se convierten en lecciones que no se reciben en una universidad, ese conocimiento sólo lo tienen los colegas.

\subsection{El estudiante}

- En estos momentos de tanta violencia intrafamiliar se tiene que ser muy paciente para tratar a los niños, hay que tener en cuenta que hay mucha contaminación social y los docentes como orientadores del proceso deben dar ejemplo de tolerancia, sinónimo de paz.

- Es muy importante en la labor del maestro la paciencia docente ya que esta es la capacidad que se debe tener de comprensión de sus estudiantes como seres únicos e irrepetibles y autó- 
nomos, como seres sociales y trascendentes. Esta es el soporte pedagógico en el aula y el mecanismo fundamental para que la práctica cotidiana trascienda.

- El docente no puede desconocer que con el Internet han surgido nuevas formas de interacción que influyen en la construcción de la identidad personal y colectiva.

- Con la trova se motiva al estudiante para que se recree, y para que utilice el tiempo libre haciendo literatura, a los niños les encanta cuando se hacen creaciones en el aula, ellos comprenden que una creación y otra, van a formar los textos.

- La poesía es otro agregado, la declamación les encanta, sobretodo aquellos versos costumbristas, jocosos y reales.

- A los niños les encanta que se les grabe; desde la escuela bien se puede descubrir valores deportivos, artísticos y culturales, al mismo tiempo brindarles oportunidades de divulgación, presentarlos en otras instituciones.

- Desde la praxis se debe tratar por mantener a los niños muy motivados y alegres en el aula, respetando sus derechos, siendo flexibles al aceptar sus sugerencias o decisiones que beneficien el grupo, haciendo del momento pedagógico un verdadero espacio de aprendizaje y de alegría.

- La praxis educativa desde el área de español, es orientada a enseñar al estudiante las cuatro habilidades comunicativas que son: leer, hablar, escribir y escuchar; ya que si el niño desarrolla estas habilidades puede descubrir un sinnúmero de experiencias que le ayudaran a alcanzar su proyecto de vida.

- Cuando se tiene la responsabilidad de ser maestro, la labor nunca termina, constantemente se piensa y se trabaja en función de los estudiantes y la manera de sacarlos adelante con buenas bases que les permita si no a todos al menos a la mayoría de ellos ser acep- tados en su comunidad y sociedad.

Para finalizar, mas no para tratar de poner un punto final, sino todo lo contrario unos suspensivos a este tema, trataré de cerrar con las apreciaciones hechas por Jorge Luis Herrera Fuentes en su documento "La didáctica del proceso docente para el desarrollo de la práctica laboral en las empresas", en el cual sostiene que: "La didáctica se puede entender como pura técnica o ciencia aplicada y como teoría o ciencia básica de la instrucción, educación o formación. Los diferentes modelos didácticos pueden ser modelos teóricos (descriptivos, explicativos, predictivos) o modelos tecnológicos (prescriptivos, normativos)...

De igual manera, se presume que la didáctica es la ciencia social que estudia el proceso docente-educativo (su objeto) orientado a la formación de las personas. Hoy se debaten en la comunidad científica, los criterios de considerar a la didáctica como una ciencia o como una rama o disciplina particular de la pedagogía. Se ha asumido el primero, el de ser la ciencia del proceso docente-educativo. En este sentido, está estrechamente ligada con la pedagogía, cuyo objeto es el proceso de formación de las personas, ya que una parte decisiva de esta formación, transcurre en la escuela y de esta disciplina se ocupa la didáctica, que se ha ido independizando de la pedagogía, sin perder el vínculo que las une" ${ }^{8}$ (HERRERA 2004)

\section{Conclusiones}

\section{El cerebro no es un vaso por llenar, sino una lámpara por encender.}

Plutarco.

Al jugar logramos aumentar el interés por el conocimiento. Unos juegos nos permiten tener idea de las aproximaciones y otros juegos nos ayudan a abstraer. Fa-

8 Idem, página 5 
cilitando así procesos de desarrollo cognitivos. En los ambientes de espontaneidad lúdica es posible que también aprendamos a comprender y transformar el mundo. Los encuentros lúdicos permiten que cada uno se exprese, imagine, acierte, sugiera, se equivoque, reconozca... con los juegos el niño relaciona, comprueba y elige. Lentamente gana confianza, voluntad y paciencia, logrando así un desarrollo natural e integral de su personalidad. El juego aparece como una de las primeras formas de expresión y creatividad con la que los niños equilibran fantasías y realidades.

Los juegos hoy en día han sido reemplazados por los juegos electrónicos y las nuevas tecnologías. Los niños de hoy navegan en el computador con personajes virtuales, formas y efectos que muchas veces los aíslan. Estos medios masivos de comunicación, pueden estar ayudando a formar una cultura homogénea, que navega constantemente por los continentes sin obligar a salir de sus domicilios a los cibernautas.

A través de la lúdica el estudiante aprende a convivir, a coexistir a partir de valores individuales y colectivos, creando así una comunidad escolar sensible, crítica y solidaria.

Es necesario aplicar nuevas formas pedagógicas que inviten al descubrimiento de las ciencias, procurar la cualidad edu- cativa que nos introduzca a una sociedad afirmativa de valores humanos.

Cuando el docente es inquieto, indaga, reflexiona y está presto a los cambios de la época acogiéndolos en su trabajo como una manera de mejorar su labor, cuando se interesa por el bienestar de sus estudiantes, y su medio familiar y social para procurar hacer un trabajo más humano, se puede decir que investiga más allá del aula.

Existe un gran interés por los docentes en generar y construir unos sujetos sociales con todas las habilidades y competencias necesarias para vivir felices y exitosos; por tal razón, la mirada de los maestros frente a la formación de hombres pensadores, situados y erguidos, está puesta hacia la formación de seres respetuosos, disciplinados, con criterio para decidir y argumentar sus opiniones y que sean felices consigo mismo.

El docente es en un ser líder constructor de sociedad, el hacedor de poetas, doctores, gobernantes, empresarios... quienes finalmente serán la prueba fehaciente, concreta, palpable de las enseñanzas recibidas de un maestro. El producto final es responsabilidad de la persona porque en últimas es el propio individuo quién decide que hacer, que camino coger y cómo explotar todo su potencial de aprendizaje.

\section{Bibliografía}

González González, Miguel (2006). Visión de filósofos y literatos sobre el devenir de la universidad contemporánea. Universidad de Manizales.

Herrera Fuentes, Jorge Luis. (2004). La didáctica del proceso docente para el desarrollo de la práctica laboral en las empresas.

Quintar, Estela B, (2006) “La Enseñanza como puente a la vida". Colección Conversaciones Didácticas, Instituto Pensamiento y Cultura En América Latina, A.C. México.
Meirieu, Philippe, (1998) Frankenstein Educador, Leaters Ediciones, Barcelona, España.

Herrera Fuentes, Jorge L, (2008) La didáctica del proceso docente para el desarrollo de la práctica laboral en las empresas, Revista electrónica 'actualidades investigativas en educación’ \#2, vol 4.

http://revista.inie.ucr.ac.cr/articulos/2-2004/archivos/didactica.pdf 